Y. Hasegawa

Nagoya Math. J.

Vol. 90 (1983), 155-173

\title{
LÉVY'S FUNCTIONAL ANALYSIS IN TERMS OF AN INFINITE DIMENSIONAL BROWNIAN MOTION III
}

\author{
YOSHIHEI HASEGAWA
}

\section{§ 0. Introduction}

The purpose of this paper is to define minimality of surfaces in an infinite dimensional space $E$ by probabilistic methods with the description of the relation between minimal surfaces and harmonic functions on the space $\boldsymbol{E}$, and to analyze purely analytic properties of a certain class of quadratic forms on the space $\boldsymbol{E}$.

We have constructed in the previous papers (Hasegawa [3], [4]) ${ }^{1}$ an infinite dimensional sequence space $\boldsymbol{E}$ :

$$
\boldsymbol{E}=\left\{x=\left(x_{1}, \cdots, x_{n}, \cdots\right) \in R^{\infty} ; \sup _{N}\left(x_{1}^{2}+\cdots+x_{N}^{2}\right) / N<\infty\right\},
$$

a system of semi-norms $\left\{\|\|_{N} ; 1 \leqslant N \leqslant \infty\right\}$ :

$$
\|x\|_{N}=\left[\left(x_{1}^{2}+\cdots+x_{N}^{2}\right) / N\right]^{1 / 2} \text { and }\|x\|_{\infty}=\varlimsup_{N \uparrow \infty}\|x\|_{N},
$$

and an infinite dimensional Brownian motion $\boldsymbol{B}=\left(\Omega, B(t, \omega), P^{x}\right)$ on the space $E$ :

$$
B(t, \omega)=\left(b_{1}(t, \omega), \cdots, b_{n}(t, \omega), \cdots\right) \in \boldsymbol{E},
$$

where $\left\{b_{n}(t, \omega) ; n \geqslant 1\right\}$ is a family of mutually independent 1-dimensional Brownian motions. The Laplacian $\triangle_{\infty}$ on the space $\boldsymbol{E}$ is defined as the infinitesimal generator of the Brownian motion $\boldsymbol{B}$ up to constant 1/2. Then we have interpreted some peculiar phenomena to P.Lévy's potential theory on the real Hilbert space $L^{2}([0,1])$ mainly through the semi-norm \|\|$_{\infty}$. In this paper we shall describe other peculiarities to his theory again with the aid of the semi-norm \|\|$_{\infty}$.

Lévy has introduced to his theory important concepts in the geometry of the space $L^{2}([0,1])$, i.e., the curvatures, in particular the mean curvature

Received May 15, 1982.

1) Without special mentions we shall use the terminologies in Hasegawa [3], [4]. 
$K$ of a surface, (cf. Lévy [1]). An analogue of such $K$, still denoted by the same symbol, will play a dominant role in this paper.

Suppose we are given a surface $S=\{x \in D ; u(x)=0\}$ by a function $u(x)$ on a domain $D$ of the space $E$. Then the mean curvature $K$ of the surface $S$ would be expected to have the following form:

$$
K=\triangle_{\infty} u /\|\operatorname{grad} u\|_{\infty},
$$

similar to the formula in the finite dimensional case. Unfortunately, there are some difficulties in defining the gradient. We are therefore interested in the correspondence between the properties $K>0, K=0, K<0$ of the surface $S$ and the ones $\triangle_{\infty} u>0, \triangle_{\infty} u=0, \triangle_{\infty} u<0$ of the function $u(x)$ on the surface $S$, respectively, (see Definition 2 and Theorem 1 ).

On the other hand, Lévy has also observed the following peculiar phenomenon to his theory: For any point $x$ of a minimal surface $S$ in the sense that $K=0$, the "uniform probability measure" on the sphere $S(x)$ around the point $x$ concentrates on the poor subset $S \cap S(x)$.

Motivated by this phenomenon, we introduce the concept of $\boldsymbol{B}$-minimality with the aid of our Brownian motion $\boldsymbol{B}$.

Definition. The surface $S$ in the domain $D(\subset E)$ with the first exit time $\tau$ is said to be $B$-minimal, if the following holds:

$$
\boldsymbol{B}_{t}(\omega) \in S \quad \text { for all } t \in[0, \tau(\omega)) \text { a.s. } P^{x}, \quad x \in S .
$$

Then we shall prove the equivalence of the minimality $(K=0)$ and the $\boldsymbol{B}$-minimality of surfaces, which contrasts with the finite dimensional cases.

Theorem 1. Let $f(x)$ be a measurable, \|\|$_{\infty}$-continuous function on a regular domain $D(\subset E)$. Then the following assertions are mutually equivalent.

1) The function $f(x)$ is harmonic on $D$.

2) For any constant $c$, the surface $S_{c}=\{x \in D ; f(x)=c\}$ is B-minimal.

It is noted that this theorem tells also the restrainted behaviour of the Brownian sample paths $B(t, \omega)$.

We can then formulate Plateau's problems in the $\boldsymbol{B}$-minimality sense, and we have the following theorem.

Theorem 3. Let $\phi(\xi)$ be a bounded, measurable, \|\|$_{\infty}$-continuous function on the surface $S$ of a strongly regular domain $D(\subset E)$ with the bounded 
exit time $\tau$. Then there exists a unique function $f(x)$ on $D$ satisfying the following conditions:

1) The function $f(x)$ is bounded, \|\|$_{\infty}$-continuous and harmonic on $D$.

2) For any point $\zeta \in S$, the function $f(x)$ converges to $\phi(\zeta)$, if $x \in D$ tends to $\zeta$ in the \|\|$_{\infty}$-sense.

As Lévy insists in his book (Lévy [1]), we see the equivalence between the Dirichlet problems and Plateau's problems, which also contrasts with the finite dimensional cases. Indeed, the surface $S_{c}=\{x \in D ; f(x)=c\}$ with $f(x)$ in Theorem 3 is $\boldsymbol{B}$-minimal by Theorem 1 and it is spanned in the contour "line" $C_{c}=\{\zeta \in S ; \phi(\zeta)=c\}$.

The role of the strong regularity property of $D$ in Theorem 3 will be explained in Section 3, (see Theorem 4), and strongly regular domains will be illustrated there, (see Theorem 5).

We now turn to the analysis of functions $f(x)$ on the space $E$, in particular infinite dimensional quadratic forms. There are requested purely analytic methods for our profound analysis of $f(x)$ on $E$, since the Brownian sample paths $B(t, \omega)$ behave in a very much restricted manner, as was seen above. Another observation on the paths is concerned with the derivatives of the function $f(x)=\|x\|_{\infty}^{2}$. The gradient of the function $f(x)$ at a point $a=\left(a_{1}, \cdots, a_{n}, \cdots\right) \in \boldsymbol{E}$ vanishes from the probabilistic point of view:

$$
\lim _{N \uparrow \infty}\left(\sum_{n=1}^{N} a_{n} b_{n}(t, \omega)\right) / N=0 \quad \text { for all } t \geqslant 0 \text { a.s. } P^{0} .
$$

Hence, for the study of the differentiation of general functions $f(x)$ on $\boldsymbol{E}$, we need not only probabilistic methods but also analytic ones. We shall therefore put more stress on the study of analytical properties of the functions $f(x)$.

To fix the idea, we shall first restrict our attention to the class of quadratic forms. Such a restriction enables us to think of a counterpart of the fact that the quadratic forms are concordant with the concepts of curvatures in the finite dimensional case.

Next we shall state the main results concerning the quadratic forms. Given a bounded sequence $\left\{\lambda_{n} ; n \geqslant 1\right\}$ of real numbers $\lambda_{n}$ with the limit $\lambda=\lim _{N \uparrow \infty}\left(\lambda_{1}+\cdots+\lambda_{N}\right) / N$ satisfying also the restriction $\lambda_{n} \geqslant \lambda_{0}$ for some constant $\lambda_{0}>0$. Then we define a function $f_{1}(x)$ on the space $E$ as follows: 


$$
f_{1}(x)= \begin{cases}\lim _{N \uparrow \infty}\left(\lambda_{1} x_{1}^{2}+\cdots+\lambda_{N} x_{N}^{2}\right) / N, & \text { if the limit exists } \\ +\infty, & \text { otherwise }\end{cases}
$$

Setting $\stackrel{\circ}{\boldsymbol{E}}=\left\{x \in \boldsymbol{E} ; f_{1}(x)<+\infty\right\}$, the complement $\boldsymbol{E}^{\prime}=\boldsymbol{E} \backslash \stackrel{\circ}{\boldsymbol{E}}$ is a \|\|$_{\infty}$-open, dense subset of $\boldsymbol{E}$, (see Theorem 6). While we shall prove (see Theorem 7):

$$
B_{t}(\omega) \in \stackrel{\circ}{E} \quad \text { for all } t \geqslant 0 \text { a.s. } P^{x}, x \in \stackrel{\circ}{E},
$$

which describes the restricted behaviour of the Brownian paths $B(t, \omega)$ topologically. This result suggests us to define a quadratic form $f_{2}(x)$ as follows:

$$
f_{2}(x)=\varlimsup_{N \uparrow \infty}\left(\lambda_{1} x_{1}^{2}+\cdots+\lambda_{N} x_{N}^{2}\right) / N, \quad x=\left(x_{1}, \cdots, x_{n}, \cdots\right) \in E,
$$

which is \|\|$_{\infty}$-continuous on the whole space $\boldsymbol{E}$, contrary to the function $f_{1}(x)$. In a similar spirit to the definition of $f_{2}(x)$, we have, (see Theorem 8 ):

$$
\varlimsup_{N \uparrow \infty}\left(\lambda_{\tau(1)} x_{1}^{2}+\cdots+\lambda_{\tau(N)} x_{N}^{2}\right) / N=\varlimsup_{N \uparrow \infty}\left(\lambda_{1} x_{\sigma(1)}^{2}+\cdots+\lambda_{N} x_{\sigma(N)}^{2}\right) / N \quad \text { on } E,
$$

where $\sigma$ denotes some permutation on the natural numbers with the inverse $\tau$. This theorem shows how the surfaces $\left\{x \in \boldsymbol{E} ; f_{2}(x)=c\right\}$ change under the permutation $\sigma$. We shall also have a criterion of harmonicity for a class of quadratic forms, (see Theorem 7).

Finally we show that the most basic quadratic form $\|x\|_{\infty}^{2}$ cannot be Fréchet differentiable at some points, which is most typical in analytical character, (see Theorem 12).

\section{$\S 1$. Harmonic functions and $B$-minimal surfaces}

In this section, we shall introduce the concept of $\boldsymbol{B}$-minimality of a surface in the space $\boldsymbol{E}$ and describe the relation between $\boldsymbol{B}$-minimal surfaces and harmonic functions.

First we recall the definition of harmonic functions on $E$. The topology induced by the semi-norms $\left\{\|\|_{N} ; 1 \leqslant N \leqslant \infty\right\}$ is called the $O_{1^{-}}$ topology, and the one induced by the semi-norms $\left\{\|\|_{N} ; 1 \leqslant N<\infty\right\}$ is called the $\mathrm{O}_{2}$-topology, respectively. We shall use the $O_{1}$-topology only, without special mentions. But the $\sigma$-algebra $\mathscr{E}$ of the space $\boldsymbol{E}$ is generated by the $O_{2}$-open subsets. Next a subset $\mathrm{A}$ of $E$ is said to be semi-bounded, if the set $\left\{\|x\|_{N} ; x \in A\right\}$ is bounded for some semi-norm \|\|$_{N},(1 \leqslant N \leqslant \infty)$.

Now we are in a position to state the following 
Definition 1. For a given domain $D(\subset E)$, we denote by $\mathscr{U}(D)$ a family of semi-bounded domains $U$ such that the closure $\bar{U}$ of $U$ is included in $D$ and that the first exit time $\tau_{U}$ from $U$ is an $\left\{\mathscr{F}_{t}\right\}$ stopping time. Then a real measurable function $f(x)$ on $D$ is said to be harmonic on the domain $D$, if for any $U \in \mathscr{U}(D)$ the following holds on $D$ :

$$
E^{x}\left[f\left(B\left(\tau_{U}\right)\right)\right]=f(x) .
$$

Next we remind the definition of $\boldsymbol{B}$-minimality.

Definition 2. For a given continuous, measurable function $u(x)$ defined on a measurable domain $D(\subset E)$, a level set $\{x \in D ; u(x)=c\}$, ( $c$ : a constant) is called a surface in the domain $D$ defined by the function $u(x)$ and denoted by $S_{u, c}$ or simply by $S_{c}$, admitting degenerated surfaces.

Definition 3. A surface $S$ in a measurable domain $D$ with the first exit time $\tau$ is said to be $\boldsymbol{B}$-minimal, if the following holds:

$$
B_{t}(\omega) \in S \quad \text { for all } t \in[0, \tau(\omega)) \text { a.s. } P^{x}, x \in S .
$$

Then, as stated in the introduction, we have the following theorem which shows the equivalence of the minimality $(K=0)$ and the $B$-minimality of surfaces.

Definition 4. We say that a nonempty open set $D$ is regular, if there exists a \|\|$_{\infty}$-continuous, measurable function $u(x)$ on $\boldsymbol{E}$ such that $D=\{x \in \boldsymbol{E} ; u(x)<0\}$ and $u(x)$ is bounded on each \|\|$_{\infty}$-ball.

Theorem 1. For a measurable, \|\|$_{\infty}$-continuous function $f(x)$ on a regular domain $D$, the following two conditions are equivalent:

1) The function $f(x)$ is harmonic on $D$.

2) For any constant $c$, the surface $S_{c}=\{x \in D ; f(x)=c\}$ is B-minimal.

Proof. It is sufficient to show the $\boldsymbol{B}$-minimality of the surfaces $S_{c}$ for the harmonic function $f(x)$. Choose a function $u(x)$ on $\boldsymbol{E}$ so as to satisfy the condition in Definition 4. Fix a point $x \in \boldsymbol{E}$. The function $u(B(t))$ is $\bigwedge_{n=1}^{\infty} \sigma\left(b_{n}(t), b_{n+1}(t), \cdots\right)$-measurable because of the \|\|$_{\infty}$-continuity, (see (0.3)). Hence we have the following with the aid of Kolmogorov's 0-1 law:

$$
u\left(B_{t}(\omega)\right)=E^{x}\left[u\left(B_{t}\right)\right] \text { a.s. } P^{x} \quad \text { for all } t \geqslant 0 .
$$


The \|\|$_{\infty}$-continuity of the paths $B(t, \omega)$ in $t$ shows the continuity of the function $u\left(B_{t}(\omega)\right)$ in $t$. Since $u(x)$ is bounded on every \|\|$_{\infty}$-bounded set, the right-hand side of (1.3) is also continuous in $t$. Hence we have the following:

$$
u\left(B_{t}(\omega)\right)=E^{x}\left[u\left(B_{t}(\omega)\right)\right] \quad \text { for all } t \geqslant 0 \text { a.s. } P^{x} .
$$

Hence there exists a function $\tau(x) \in[0, \infty]$ on the space $E$ such that

$$
\tau(\omega)=\tau(x) \text { a.s. } P^{x}, \quad(x \in E),
$$

where $\tau(\omega)$ denotes the first exit time from the domain $D$. Next we fix a point $a \in D$ and $t_{0}, T$ such that $0<t_{0}<T<\tau(a)$. Then, by (1.4) we have a constant $\delta$ :

$$
\max \left\{u\left(B_{t}(\omega)\right) ; 0 \leqslant t \leqslant T\right\}=-\delta<0 \text { a.s. } P^{a} .
$$

Therefore

$$
B_{t}(\omega) \in G_{\delta} \quad \text { for all } t \in[0, T] \text { a.s. } P^{a} \text {, }
$$

where

$$
G_{\delta}=\{x \in E ; u(x)<-\delta / 2\} .
$$

Then the first exit time $\tilde{\tau}_{\delta}(\omega)$ from the measurable, \|\|$_{\infty}$-open subset $\tilde{G}_{\delta}$ :

$$
\tilde{G}_{\delta}=G_{\delta} \cap\left\{x \in E ;\|x-a\|_{\infty}<\sqrt{t_{0}}\right\}
$$

is deterministic and the formula (1.5) shows

$$
\tilde{\tau}_{\delta}(\omega)=t_{0} \text { a.s. } P^{a} \text {. }
$$

Noticing the closure $\overline{\tilde{G}}_{\delta}$ of $\tilde{G}_{\delta}$ is included in $D$, we have consequently the following equality by the harmonicity of $f(x)$ :

$$
E^{a}\left[f\left(B\left(t_{0}\right)\right)\right]=E^{a}\left[f\left(B\left(\tilde{\tau}_{\delta}\right)\right)\right]=f(a) .
$$

In a quite similar manner to the derivation of (1.4), we have therefore the following:

$$
f\left(B_{t}(\omega)\right)=E^{a}\left[f\left(B_{t}\right)\right]=f(a) \quad \text { for all } t \in[0, \tau(a)) \text { a.s. } P^{a} \text {. Q.E.D. }
$$

Consequently the Brownian paths $B(t, \omega)$ move almost surely on the surface $S_{c}=\{x \in D ; f(x)=c\}$ defined by the harmonic function $f(x)$ on the domain $D$.

Here we shall describe some properties of the boundary of the regular domain $D$ in Definition 4 . The boundary of $D$ with respect to the $O_{1}$ - 
-topology coincides with the one of $D$ with respect to the \|\|$_{\infty}$-topology, and, however, may not be measurable. We shall forward denote by $\partial D$ and $S$ the boundary of $D$ and the surface $\{x \in E ; u(x)=0\}$, respectively. Then it is easily seen that $\partial D \subset S$, and further the following theorem holds.

THEOREM 2. The boundary $\partial D$ of a nonempty regular domain $D$ coincides with the surface $S$, provided that the function $u(x)$ defining $D=\{x$ $\in E ; u(x)<0\}$ is convex on $\boldsymbol{E}$.

Proof. It is sufficient to prove $S \subset \partial D$. Take points $\zeta \in S$ and $a \in D$, and set $f(r)=u(r(\zeta-a)+a),(r \geqslant 0)$. Then it can be seen that $f(r)$ is convex and $f(0)=u(a)<0, f(1)=0$, which shows that $r=1$ is the unique solution of the equation $f(r)=0$. Since $r(\zeta-a)+a$ belongs to $D$ for $r \in[0,1)$ and converges to $\zeta$ as $r \uparrow 1$, the proof is now complete.

Lastly we note that the first exit time $\tau$ of a regular domain is deterministic.

\section{§2. Plateau's problems and the Dirichlet problems}

In this section, we shall formulate Plateau's problems under the identification of the minimality $(K=0)$ and the $B$-minimality.

Definition 5. A nonempty regular domain $D(\subset E)$ is said to be strongly regular, provided that the function $u(x)$ defining the domain $D$ $=\{x \in E ; u(x)<0\}$ in Definition 4 is strictly subharmonic on the surface $S=\{x \in E ; u(x)=0\}$ in the following sense:

$$
\int u(\zeta+r \xi) \mu(d \xi)>0 \quad \text { for any } r>0 \text { and } \zeta \in S,
$$

where $\mu$ stands for the standard Gaussian white noise.

Remark. A finite intersection of (resp., strongly) regular domains is (resp., strongly) regular.

Now we are ready to state our realization of Lévy's insistence that Plateau's problems and the Dirichlet problems are mutually equivalent in his theory.

TheOREm 3. We are given a strongly regular domain $D(\subset E)$ with the bounded exit time $\tau$. Then for a bounded, measurable, \|\|$_{\infty}$-continuous function $\phi(\xi)$ on the surface $S$ of $D$, the function $f(x)$ 


$$
f(x)=E^{x}\left[\phi\left(B_{\imath}\right)\right], \quad(x \in D),
$$

is the unique one satisfying the following two conditions:

1) The function $f(x)$ is bounded, \|\|$_{\infty}$-continuous and harmonic on $D$.

2) For any point $\zeta \in S$, the function $f(x)$ converges to $\phi(\zeta)$, if $x \in D$ tends to $\zeta$ in the \|\|$_{\infty}$-sense.

Proof. First choose a function $u(x)$ on $E$ such that $D=\{x \in E ; u(x)$ $<0\}$ and the function $u(x)$ satisfies the condition of Definition 5 . Set

$$
v(x, t)=\int u(x+\sqrt{t} \xi) \mu(d \xi) \quad \text { for } t \geqslant 0 \text { and } \quad x \in \boldsymbol{E} .
$$

Then in a quite similar way to the derivation of (1.4), we have the following:

$$
u\left(B_{t}(\omega)\right)=v(x, t) \quad \text { for all } t \geqslant 0 \text { a.s. } P^{x}, \quad(x \in E) .
$$

Hence there exists a bounded function $\tau(x) \in[0, \infty)$ on $D$ such that

$$
\tau(\omega)=\tau(x) \text { a.s. } P^{x},
$$

and $\tau(x)$ is a solution of the following equation:

$$
v(x, t(x))=0, \quad(x \in D) .
$$

Suppose now we have a solution $t(x)$ of (2.5) other than $\tau(x),(x \in D$; fixed). Then by (2.4), it holds that

$$
u(B(t(x)))=u(B(\tau(x)))=0 \text { a.s. } P^{x} .
$$

Hence observing the mutual independence of $B(t(x))-B(\tau(x))$ and $B(\tau(x))$, we get the following contradiction from the strict subharmonicity of $u(x)$ at $y=B(\tau(x)) \in S$ :

$$
\begin{aligned}
0 & =E^{x}[u(B(t(x))) \mid \sigma(B(s) ; 0 \leqslant s \leqslant \tau(x))] \\
& =\int u(y+\sqrt{t(x)-\tau(x)} \xi) \mu(d \xi)>0 \text { a.s. } P^{x} .
\end{aligned}
$$

Therefore $\tau(x),(x \in D)$ is the unique solution of the equation (2.5). Namely it holds that

$$
v(x, t)<0 \quad \text { for all } t<\tau(x) \text {, and } v(x, t)>0 \text { for all } t>\tau(x) .
$$

Hence we obtain

$$
\{x \in D ; \tau(x)>t\}=\{x \in D ; v(x, t)<0\} \quad \text { for all } t \geqslant 0,
$$


which implies the measurability of the function $\tau(x)$.

$$
\text { If } \begin{aligned}
\left\|x_{n}-x\right\|_{\infty} \rightarrow 0, \quad \tau\left(x_{n}\right) \rightarrow t \quad \text { as } n \uparrow \infty, \quad\left(x_{n}, x \in D\right), \text { then } \\
v(x, t)=\lim _{n \uparrow \infty} v\left(x_{n}, \tau\left(x_{n}\right)\right)=0 .
\end{aligned}
$$

Thus the \|\|$_{\infty}$-continuity of $\tau(x)$ on $D$ also follows from the uniqueness of the solution $t(x)$ of (2.5).

Next assume that there exist $t>0$, a point $\zeta \in S$ and a sequence $\left\{x_{n} ; n \geqslant 1\right\}$ of points $x_{n} \in D$ such that $\left\|x_{n}-\zeta\right\|_{\infty} \rightarrow 0, \tau\left(x_{n}\right) \rightarrow t$ as $n \uparrow \infty$. Then it holds that

$$
u(\zeta+B(t))=\lim _{n \uparrow \infty} u\left(x_{n}+B\left(\tau\left(x_{n}\right)\right)\right)=0 \text { a.s. } P^{0} .
$$

Hence the strict subharmonicity of $u(x)$ on $S$ induces the following contradiction:

$$
0=\int u(\zeta+\sqrt{t} \xi) \mu(d \xi)>0,
$$

which shows that $\tau\left(x_{n}\right) \rightarrow 0$ as $n \uparrow \infty$, if $\left\|x_{n}-\zeta\right\|_{\infty} \rightarrow 0$ as $n \rightarrow \infty$. Since the function $\phi(\xi)$ on the measurable surface $S$ is \|\|$_{\infty}$-continuous, measurable, and the Brownian motion $\boldsymbol{B}$ is strong Markov, it is now easily seen that the function $f(x)$

$$
f(x)=E^{x}\left[\phi\left(B_{\tau}\right)\right]=\int \phi(x+\sqrt{\tau(x)} \xi) \mu(d \xi), \quad(x \in D),
$$

satisfies the conditions 1), 2) of this theorem.

Finally, assuming that there is another function $g(x)$ satisfying the conditions 1), 2), we shall prove $h(x)=f(x)-g(x)$ must be zero. Now fix a point $a \in D$ and define regular domains $D_{n}=\{x \in E ; u(x)<-1 / n\}$, $(n \geqslant 1)$. The first exit times $\tau_{n}$ from $D_{n}$ are deterministic and have the limit $\tau_{0}=\lim _{n \uparrow \infty} \tau_{n}(\leqslant \tau<\infty)$. Then it holds that $v\left(a, \tau_{0}\right)=0$. Hence the uniqueness of the solution $t(a)$ of (2.5) shows $\lim _{n \uparrow \infty} \tau_{n}=\tau(a)$ a.s. $P^{a}$, and we obtain the following:

$$
\lim _{n \uparrow \infty} h\left(B\left(\tau_{n}\right)\right)=0 \text { a.s. } P^{a} .
$$

Therefore the harmonicity of the function $h(x)$ on $D$ gives

$$
h(a)=E^{a}\left[h\left(B\left(\tau_{n}\right)\right)\right]=\lim _{n \uparrow \infty} E^{a}\left[h\left(B\left(\tau_{n}\right)\right)\right]=0
$$

with the aid of Lebesgue's dominated convergence theorem. That is, 


$$
f(x)=g(x) \quad \text { on the domain } D .
$$

Q.E.D.

Remark. If the $D$ is \|\|$_{\infty}$-bounded, the exit time $\tau(x)$ is bounded on $D$.

\section{§ 3. Strongly regular domains}

In this section, we shall explain the necessity of the strong regularity condition on the domain $D$ to have Theorem 3 , and give some (strictly) subharmonic functions $u(x)$ associated with the $D$.

Definition 6. Let $\left\{\mu_{x} ; x \in D\right\}$ be the harmonic measures relative to a regular domain $D$ and $x \in D$ :

$$
\mu_{x}(A)=P^{x}(B(\tau) \in A), \quad(A \subset S),
$$

where $\tau$ and $S$ denote the first exit time from $D$ and the surface of $D$, respectively. Then a boundary point $\zeta \in \partial D$ is said to be irregular, if there exists a bounded, measurable, \|\|$_{\infty}$-continuous function $\phi(\xi)$ on $S$ such that the function $f(x)=\int \phi(\xi) \mu_{x}(d \xi)$ on $D$ does not converge to $\phi(\zeta)$ as $x \in D$ tends to $\zeta$ in the \|\|$_{\infty}$-sense.

Theorem 4. Let $D$ be a regular domain with the first exit time $\tau$ defined by a function $u(x)$ on $E$ such that $D=\{x \in E: u(x)<0\}$. Suppose we are given a boundary point $\zeta \in \partial D$ and $a$ ball $U=\left\{x \in E ;\|x-\zeta\|_{\infty}<r\right\}$, $(r>0)$ with the first exit time $\tau_{U}$ such that $u(x)$ is superharmonic on $U$ in the following sense:

$$
E^{x}\left[u\left(B_{t}\right)\right] \leqslant u(x) \quad \text { for all } t<\tau_{U} \text { a.s. } P^{x}, \quad(x \in U) .
$$

Then the boundary point $\zeta$ is irregular.

Proof. First fix a point $a \in D \cap U$ such that $\|a-\zeta\|_{\infty}<\sqrt{3} r / 2$. Then the superharmonicity of $u(x)$ on $U$ gives:

$$
u\left(B_{t}(\omega)\right)=E^{a}\left[u\left(B_{t}\right)\right] \leqslant u(a)<0 \quad \text { for all } t<\tau_{U} \text { a.s. } P^{a},
$$

where $\tau_{U}(\omega)=r^{2}-\|a-\zeta\|_{\infty}^{2} \geqslant r^{2} / 4$ a.s. $P^{a}$. Since $\tau$ is deterministic, therefore, we obtain:

$$
\|B(\tau)-\zeta\|_{\infty}^{2}=\|\zeta-a\|_{\infty}^{2}+\tau \geqslant r^{2} / 4 \text { a.s. } P^{a} .
$$

Now it is clear that $\zeta \in \partial D$ is irregular in the above-mentioned sense.

Q.E.D. 
EXAMPLE. Set

$$
D=\left\{x \in E ;\|x\|_{\infty}<1,\langle x, \xi\rangle_{\infty}>0\right\},
$$

where $\xi \in \boldsymbol{E}$ with $\|\xi\|_{\infty}=1$ and $4\langle x, \xi\rangle_{\infty}=\|x+\xi\|_{\infty}^{2}-\|x-\xi\|_{\infty}^{2}$. Then the boundary point $\zeta \in \partial D$ such that $\|\zeta\|_{\infty}\left\langle 1,\langle\zeta, \xi\rangle_{\infty}=0\right.$ is irregular.

Observing the proof of the above theorem, it is reasonable to assume the strict subharmonicity of $u(x)$ on $S$ for the sake of the regularity of the boundary.

Next we give some (strictly) subharmonic functions $u(x)$ on $\boldsymbol{E}$.

TheORem 5. We are given a sequence $\left\{\lambda_{n} ; n \geqslant 1\right\}$ of real numbers $\lambda_{n}$ with $\lambda_{\max } \geqslant \lambda_{n} \geqslant \lambda_{\min }>0$ and a continuous, subharmonic ${ }^{1)}$ function $\phi(s)$ on $R^{d}$ such that

$$
|\phi(s)-\phi(t)| \leqslant c|t-s|^{\alpha}(1+|s|+|t|)^{2-\alpha}, \quad\left(s, t \in R^{d}\right),
$$

with constants $c, \alpha,(c>0,0<\alpha \leqslant 1)$. Then the function $u(x)$

$$
u(x)=\varlimsup_{N \uparrow \infty}\left[\sum_{n=1}^{N} \lambda_{n} \phi\left(x_{(n-1) d+1}, \cdots, x_{n d}\right)\right] / N, \quad x=\left(x_{1}, \cdots, x_{n} \cdots\right) \in \boldsymbol{E},
$$

is \| $\|_{\infty}$-continuous and bounded on every \| $\|_{\infty}$-bounded set:

$$
|u(x)-u(y)| \leqslant 3 c d \lambda_{\max }\|x-y\|_{\infty}^{\alpha}\left(1+\|x\|_{\infty}^{2}+\|y\|_{\infty}^{2}\right)^{1-\alpha / 2}, \quad(x, y \in E),
$$

and subharmonic on $\boldsymbol{E}$ in the following sense:

$$
E^{x}\left[u\left(B_{t}\right)\right] \geqslant u(x) \quad \text { for all } t \geqslant 0, \quad x \in \boldsymbol{E} .
$$

Furthermore, if the $\phi(s)$ is not harmonic on $R^{d}$, then $u(x)$ is strictly subharmonic on $\boldsymbol{E}$ in the following sense:

$$
E^{x}\left[u\left(B_{t}\right)\right]>u(x) \quad \text { for all } t>0 \text { and } x \in \boldsymbol{E} .
$$

Proof. First we shall prove the estimate (3.5). Put $\hat{x}_{n}=\left(x_{(n-1) d+1}\right.$, $\left.\cdots, x_{n d}\right)$ and $\hat{y}_{n}=\left(y_{(n-1) d+1}, \cdots, y_{n d}\right),(n \geqslant 1)$ for $x=\left(x_{1}, \cdots, x_{n}, \cdots\right) \in \boldsymbol{E}$ and $y=\left(y_{1}, \cdots, y_{n}, \cdots\right) \in \boldsymbol{E}$, respectively. Then we have the following inequality:

$$
\begin{gathered}
\left|\frac{1}{N} \sum_{n=1}^{N} \lambda_{n} \phi\left(\hat{x}_{n}\right)-\frac{1}{N} \sum_{n=1}^{N} \lambda_{n} \phi\left(\hat{y}_{n}\right)\right| \leqslant \frac{c \lambda_{\max }}{N} \sum_{n=1}^{N}\left|\hat{x}_{n}-\hat{y}_{n}\right|^{\alpha}\left(1+\left|\hat{x}_{n}\right|+\left|\hat{y}_{n}\right|\right)^{2-\alpha} \\
\leqslant c \lambda_{\max }\left(\frac{1}{N} \sum_{n=1}^{N}\left|\hat{x}_{n}-\hat{y}_{n}\right|^{2}\right)^{\alpha / 2}\left(\frac{1}{N} \sum_{n=1}^{N}\left(1+\left|\hat{x}_{n}\right|+\left|\hat{y}_{n}\right|\right)^{2}\right)^{1-\alpha / 2}
\end{gathered}
$$

1) The terms of harmonicity, subharmonicity and superharmonicity of functions on $R^{d}$ are understood according to Port-Stone [5]. 
with the aid of Hölder's inequality. Hence by limiting $N \uparrow \infty$, we obtain the estimate (3.5). Next we set $f_{t}(s)=\hat{E}^{s}[\phi(b(t))]-\phi(s), \quad\left(s \in R^{d}, t \geqslant 0\right)$, where $\left\{\hat{P}^{s}, b(t)\right\}$ stands for the $d$-dimensional Brownian motion. Fix $t \geqslant 0$ and $x=\left(x_{1}, \cdots, x_{n}, \cdots\right) \in \boldsymbol{E}$ and choose a strictly increasing sequence $\left\{N_{k} ; k \geqslant 1\right\}$ of integers $N_{k}$ such that $N_{k} \uparrow \infty$ as $k \uparrow \infty$ and

$$
\lim _{k \uparrow \infty}\left[\sum_{n=1}^{N_{k}} \lambda_{n} \phi\left(\hat{x}_{n}\right)\right] / N_{k}=u(x) .
$$

Then, noticing $\|B(t, \omega)\|_{\infty}^{2}=t+\|x\|_{\infty}^{2}$ for all $t \geqslant 0$ a.s. $P^{x}$, we have:

$$
\begin{aligned}
\infty & >E^{x}\left[u\left(B_{t}\right)\right] \geqslant \varlimsup_{N \uparrow \infty}\left[\left(\sum_{n=1}^{N} \lambda_{n}\left(f_{t}\left(\hat{x}_{n}\right)+\phi\left(\hat{x}_{n}\right)\right)\right) / N\right] \\
& \geqslant \varlimsup_{k \uparrow \infty}\left[\left(\sum_{n=1}^{N_{k}} \lambda_{n} f_{t}\left(\hat{x}_{n}\right)\right) / N_{k}\right]+u(x) .
\end{aligned}
$$

Hence the subharmonicity of $\phi(s)$ shows the one of $u(x)$ on $E$. Moreover suppose that $E^{x}\left[u\left(B_{t}\right)\right]=u(x)$ at a point $x \in E$ and a $\tilde{t}>0$. Then, by (3.8), it holds that

$$
\begin{aligned}
0 & =E^{x}\left[u\left(B_{\tilde{t}}\right)\right]-u(x)=\varlimsup_{k \uparrow \infty}\left[\left(\sum_{n=1}^{N_{k}} \lambda_{n} f_{\tilde{t}}\left(\hat{x}_{n}\right)\right) / N_{k}\right] \\
& \geqslant \lambda_{\min } \rho_{r} \varlimsup_{\lim _{\uparrow}}^{\#}\left\{n \leqslant N_{k} ;\left|\hat{x}_{n}\right| \leqslant \sqrt{d} r\right\} / N_{k} \geqslant 0,
\end{aligned}
$$

where $\rho_{r}=\min \left\{f_{\hat{t}}(s) ;|s| \leqslant \sqrt{d} r\right\},(r>0)$. Here assume that the $\phi(s)$ is not harmonic on $R^{d}$. Then there exist a point $a \in R^{d}$ and an $r_{0}>0$ such that

$$
\int \phi\left(a+r_{0} \xi\right) \sigma(d \xi)>\phi(a)
$$

where $\sigma$ denotes the uniform probability measure on the unit sphere of $R^{d}$. We can, therefore, see that $f_{t}(s)>0$ for all $t>0, s \in R^{d}$, which gives $\rho_{r}>0$. Hence we have the following contradiction:

$$
d\|x\|_{\infty}^{2} \geqslant \varlimsup_{k \uparrow \infty} \frac{1}{N_{k}} \sum_{n=1}^{N_{k}}\left|\hat{x}_{n}\right|^{2} \geqslant d r^{2} \quad \text { for any } r>0 .
$$

Consequently $u(x)$ is strictly subharmonic on $\boldsymbol{E}$.

Q.E.D.

Remark. In the above theorem, if the $\phi(s)$ is convex on $R^{d}$, then the $u(x)$ is convex on $E$. Hence the domain $D=\{x \in E ; u(x)<c\}$, (c; a constant) is regular and the boundary $\partial D$ coincides with the surface $S=\{x \in E ; u(x)$ $=c\}$, (if $D$ is nonempty), (see Theorem 2). 
For example, the function $u_{\alpha}(x),(1 \leqslant \alpha \leqslant 2)$,

$$
u_{\alpha}(x)=\varlimsup_{N \uparrow \infty}\left(\left|x_{1}\right|^{\alpha}+\cdots+\left|x_{N}\right|^{\alpha}\right) / N, \quad x=\left(x_{1}, \cdots, x_{n}, \cdots\right) \in \boldsymbol{E},
$$

is \|\|$_{\infty}$-continuous, strictly subharmonic and bounded on every \|\|$_{\infty}$ bounded set, and it holds that $\partial D_{\alpha}=S_{\alpha}$, where $D_{\alpha}=\left\{x \in E ; u_{\alpha}(x)<1\right\}$ and $S_{\alpha}=\left\{x \in E ; u_{\alpha}(x)=1\right\}$. Although $D_{\alpha},(1 \leqslant \alpha<2)$ is not \|\|$_{\infty}$-bounded, there exists a constant $M_{\alpha}>0$ such that $\tau_{\alpha}(\omega) \leqslant M_{\alpha}$ a.s. $P^{x},\left(x \in D_{\alpha}\right)$, where $\tau_{\alpha}$ denotes the first exit time from $D_{\alpha}$.

Thus we can obtain a lot of \|\|$_{\infty}$-continuous, strictly subharmonic functions bounded on every \|\|$_{\infty}$-bounded subset of $\boldsymbol{E}$ and equivalently, of strongly regular domains.

\section{§4. Quadratic forms and transformation groups}

Our analysis established in Sections $1 \sim 3$ depends heavily on the probabilistic methods. In this section we shall put stress on study of purely analytical properties of quadratic forms on the space $\boldsymbol{E}$.

To begin with, we introduce the following two functions $f_{1}(x), f_{2}(x)$ :

$$
f_{1}(x)= \begin{cases}\lim _{N \uparrow \infty}\left[\lambda_{1} x_{1}^{2}+\cdots+\lambda_{N} x_{N}^{2}\right] / N, & \text { if the limit exists } \\ +\infty, & \text { otherwise }\end{cases}
$$

with the restriction:

$$
\lambda_{\max } \geqslant \lambda_{n} \geqslant \lambda_{\min }>0
$$

and put

$$
\stackrel{\circ}{\boldsymbol{E}}=\left\{x \in \boldsymbol{E} ; f_{1}(x)<+\infty\right\}, \quad \boldsymbol{E}^{\prime}=\boldsymbol{E} \backslash \stackrel{\circ}{\boldsymbol{E}} .
$$

Then our first assertion is the following

THEOREM 6.

1) The function $f_{1}(x)$, (resp., $\left.f_{2}(x)\right)$ is \|\|$_{\infty}$-continuous on $\stackrel{\circ}{E}$, (resp., on $\left.E\right)$.

2) The set $\boldsymbol{E}^{\prime}$ is \|\|$_{\infty}$-open, and dense in $\boldsymbol{E}$ with respect to the $O_{1^{-}}$ topology.

Proof. We shall prove the denseness of $\boldsymbol{E}^{\prime}$ in the space $\boldsymbol{E}$. Take a point $a=\left(a_{1}, \cdots, a_{n}, \cdots\right) \in \stackrel{\circ}{\boldsymbol{E}}$ such that $\|a\|_{\infty}>0$, a sequence $\left\{N_{k} ; k \geqslant 0\right\}$ of integers $N_{k}$ such that $N_{k} \uparrow \infty, N_{k} / N_{k-1} \rightarrow \infty$ as $k \uparrow \infty$, and $N_{0}=0$. Next put 


$$
c_{n}=\left\{\begin{array}{rl}
-a_{n} & \text { if } N_{2 k-1}<n \leqslant N_{2 k} \\
a_{n} & \text { if } N_{2 k}<n \leqslant N_{2 k+1}
\end{array} .\right.
$$

Then, noticing the following:

$$
\lim _{k \uparrow \infty}\left[\lambda_{1} a_{1}^{2}+\cdots+\lambda_{N_{k}} a_{N_{k}}^{2}\right] / N_{k}=f_{1}(a) \geqslant \lambda_{\min }\|a\|_{\infty}^{2}>{ }_{i}^{r} 0,
$$

we have:

$$
\lim _{k \uparrow \infty}\left[\sum_{n=1}^{N_{2 k}} \lambda_{n} a_{n} c_{n}\right] / N_{2 k}=-f_{1}(a)<0, \quad \lim _{k \uparrow \infty}\left[\sum_{n=1}^{N_{2 k-1}} \lambda_{n} a_{n} c_{n}\right] / N_{2 k-1}=f_{1}(a)>0 .
$$

Therefore we have points $b_{\varepsilon}=a+\varepsilon c \in E^{\prime},(\varepsilon>0)$ and $\lim _{\varepsilon \downarrow 0}\left\|b_{\varepsilon}-a\right\|_{p}=0$ for $p \in\{1,2, \cdots, \infty\}$. For a point $a \in \dot{E}$ with $\|a\|_{\infty}=0$ the proof is immediate. The proof of the other assertion is straightforward. Q.E.D.

For the sake of the continuity on the whole space $E$ of "quadratic forms", we prefer the following

Definition 7. The function $f(x)$

$$
f(x)=\varlimsup_{N \dagger \infty}\left[\lambda_{1} x_{1}^{2}+\cdots+\lambda_{N} x_{N}^{2}\right] / N, \quad x=\left(x_{1}, \cdots, x_{n}, \cdots\right) \in E,
$$

on the space $\boldsymbol{E}$ with bounded coefficients $\left\{\lambda_{n}\right\}$ is called a quadratic form of diagonal type.

Then the quadratic form $f(x)$ is measurable \|\|$_{\infty}$-continuous on $\boldsymbol{E}$. Moreover we have the following

TheOREm 7. Assume the existence of the limit

$$
\lambda=\lim _{N \uparrow \infty}\left(\lambda_{1}+\cdots+\lambda_{N}\right) / N .
$$

Then it holds that

$$
f\left(B_{t}(\omega)\right)=f(x)+\lambda t \quad \text { for all } t \geqslant 0 \text { a.s. } P^{x}, \quad(x \in E) .
$$

In addition, we have

$$
\lim _{N \uparrow \infty} \frac{1}{N} \sum_{n=1}^{N} \lambda_{n}\left(b_{n}(t, \omega)-b_{n}(s, \omega)\right)^{2}=\lambda|t-s| \quad \text { for all } t, s \geqslant 0 \text { a.s. } P^{x},
$$

$(x \in E)$, provided that $\lambda_{n} \geqslant 0,(n \geqslant 1)$.

Proof. The proof proceeds in the same way as the ones of Proposition 1.3, Theorem 1.4 of Hasegawa [3].

Q.E.D.

If the sequence $\left\{\lambda_{n}\right\}$ satisfies the condition (4.3) and has the limit $\lambda$ 
(4.6), the formula (4.7) says that $B_{t}(\omega) \in \stackrel{\circ}{E}$ for all $t \geqslant 0$ a.s. $P^{x},(x \in \mathscr{E})$. Consequently Theorem 6 describes the restricted behaviour of the Brownian paths $B(t, \omega)$ topologically. From (4.7) we have also $\triangle_{\infty} f(x)=2 \lambda$, which can be rewritten as follows:

$$
\triangle_{\infty} f(x)=\lim _{N \dagger \infty} \sum_{j=1}^{N}\left(\partial^{2} / \partial x_{j}^{2}\right)\left[\left(\sum_{n=1}^{N} \lambda_{n} x_{n}^{2}\right) / N\right] .
$$

This is concordant with the finite dimensional construction method, (see Sections 1 and 2 of Hasegawa [3]). Moreover $f(x)$ is harmonic (resp., strictly subharmonic) on $E$, iff $\lambda=0$, (resp., $\lambda>0$ ). Analogous phenomena can be observed in the case of quadratic forms with blockwise interaction matrices A, (see Theorem 10).

Now we shall introduce a transformation group $G_{0}=\left\{g_{\sigma} ; \sigma\right\}$. Fix a sequence $\left\{n_{k} ; k \geqslant 1\right\}$ of integers $n_{k}$ such that

$$
2<n_{1}<\cdots<n_{k}<\cdots \text {, and } n_{k+1} / n_{k} \rightarrow 1 \text { as } k \uparrow \infty .
$$

Definition 8. A transformation $g$ of the space $\boldsymbol{E}$ onto itself is called a blockwise permutation associated with the sequence $\left\{n_{k}\right\}$, if there exists a permutation $\sigma$ of the set $\{1,2,3, \cdots\}$ which is also a permutation of each block $\left\{n_{k}+1, n_{k}+2, \cdots, n_{k+1}\right\}$ for each $k \geqslant 1$, and $g=g_{\sigma}$ :

$$
\left(g_{\sigma} x\right)_{n}=x_{\sigma(n)} \quad \text { for } x=\left(x_{1}, \cdots, x_{n}, \cdots\right) \in E .
$$

The permutation $\sigma$ itself is called a blockwise permutation also.

For a blockwise permutation $\sigma$ with the inverse $\tau$ and bounded coefficients $\left\{\lambda_{n}\right\}$, define a quadratic form $f_{\sigma}(x)$ as follows:

$$
f_{\sigma}(x)=\varlimsup_{N \uparrow \infty} \frac{1}{N} \sum_{n=1}^{N} \lambda_{\tau(n)} x_{n}^{2}, \quad x=\left(x_{1}, \cdots, x_{n}, \cdots\right) \in E .
$$

Then we have the following invariance theorem.

THEOREM 8. Fix a blockwise permutation $\sigma$ with the inverse $\tau$.

1) For a bounded sequence $\left\{\lambda_{n}\right\}$ with the limit $\lambda$, (4.6), it holds that

$$
\lambda=\lim _{N \dagger \infty}\left(\lambda_{\tau(1)}+\cdots+\lambda_{\tau(N)}\right) / N .
$$

2) For a bounded sequence $\left\{\lambda_{n}\right\}$ with a definite sign, it holds that:

$$
f_{\sigma}(x)=f\left(g_{\sigma} x\right),
$$

$$
\lim _{N \uparrow \infty}\left(\lambda_{\tau(1)} x_{1}^{2}+\cdots+\lambda_{\tau(N)} x_{N}^{2}\right) / N=\lim _{N \uparrow \infty}\left(\lambda_{1} x_{\sigma(1)}^{2}+\cdots+\lambda_{N} x_{\sigma(N)}^{2}\right) / N .
$$


Here the equality (4.14) means that if one of the both limits exists, so does the other and the equality holds.

3) For a bounded sequence $\left\{\lambda_{n}\right\}$ with mixed signs, the equalities (4.13), (4.14) hold for points $x \in \boldsymbol{E}$ such that $\lim _{N \uparrow \infty}\|x\|_{N}$ exist.

Proof. First we prove the equality (4.13) in case of $\lambda_{n} \geqslant 0,(n \geqslant 1)$. Fix a point $x \in E$. For an integer $N$ such that $n_{k}<N \leqslant n_{k+1}$, we put $f_{N}(x)=\left(\lambda_{1} x_{1}^{2}+\cdots+\lambda_{N} x_{N}^{2}\right) / N$ and choose an integer $q_{k} \in\left\{n_{k}+1, \cdots, n_{k+1}\right\}$ such that

$$
f_{q_{k}}\left(g_{\sigma} x\right)=\max \left\{f_{n_{k}+1}\left(g_{\sigma} x\right), \cdots, f_{n_{k+1}}\left(g_{\sigma} x\right)\right\} .
$$

Then we have the following:

$$
\left(\lambda_{\tau(1)} x_{1}^{2}+\cdots+\lambda_{\tau(N)} x_{N}^{2}\right) / N \leqslant\left(q_{k+1} / N\right) f_{q_{k+1}}\left(g_{\sigma} x\right),
$$

which induces:

$$
f_{\sigma}(x) \leqslant f\left(g_{\sigma} x\right) .
$$

Similarly we have the converse inequality, which proves (4.13) together with (4.17). The proof of (4.13) in the case of $\lambda_{n} \leqslant 0,(n \geqslant 1)$ and the one of the other assertion are now clear. Q.E.D.

The equality (4.12) shows the harmonicity-preserving property of the blockwise permutation $g_{\sigma}$ : The harmonicity of $f(x)$, (4.5) with the limit $\lambda=0$ implies the one of the quadratic form $f_{\sigma}(x)$.

From now on, we shall extend the results obtained for quadratic forms of diagonal type to a little larger class of quadratic forms.

First of all, fix a sequence $\left\{n_{k}\right\}$ satisfying the condition (4.9). Then an infinite dimensional symmetric matrix $A=\left(a_{i, j}\right)$ is called a blockwise interaction matrix associated with the sequence $\left\{n_{k}\right\}$, if the matrix $A$ has the following form:

$$
A=A_{1} \oplus A_{2} \oplus \cdots \oplus A_{n} \oplus \cdots,
$$

where each matrix $A_{k},(k \geqslant 1)$ is a real symmetric $\left(n_{k}-n_{k-1}\right) \times\left(n_{k}-n_{k-1}\right)$ matrix and the set of the eigenvalues of matrices $\left\{A_{k}\right\}$ is bounded.

Definition 9. The following function $f(x)$ defined on the space $\boldsymbol{E}$

$$
f(x)=\varlimsup_{N \uparrow \infty} \frac{1}{N} \sum_{i, j=1}^{N} a_{i, j} x_{i} x_{j} \quad \text { for } x=\left(x_{1}, \cdots, x_{n}, \cdots\right) \in E
$$

is called a quadratic form with the blockwise interaction matrix $A$. 
ThEOREM 9. The quadratic form $f(x)$ with the blockwise interaction matrix $A$ is \|\|$_{\infty}$-continuous on the space $\boldsymbol{E}$.

Proof. Since the eigenvalues of the matrices $\left\{A_{k} ; k \geqslant 1\right\}$ are bounded, we can easily prove the theorem.

Q.E.D.

Next we have an extention of Theorem 7.

THEOREM 10. Under the existence of the limit $\lambda=\lim _{N \uparrow \infty}\left(a_{1,1}+\cdots\right.$ $\left.+a_{N, N}\right) / N$

$$
f\left(B_{t}(\omega)\right)=f(x)+\lambda t \quad \text { for all } t \geqslant 0 \text { a.s. } P^{x}, \quad(x \in E) .
$$

Proof. The symmetry of the matrices $\left\{A_{k} ; k \geqslant 1\right\}$ and the rotation invariance of the finite dimensional Brownian motions reduce the proof to the one of Theorem 7 .

Q.E.D.

Definition 10. We denote by $G_{1}$ the following group:

$$
G_{1}=O\left(n_{1}\right) \oplus O\left(n_{2}-n_{1}\right) \oplus \cdots \oplus O\left(n_{k}-n_{k-1}\right) \oplus \cdots,
$$

and call an element $g \in G_{1}$ a blockwise rotation.

Remark. $\|g x\|_{\infty}=\|x\|_{\infty}$ for all $g \in G_{1}, x \in E$.

Next we define another blockwise interaction matrix $B=\left(b_{i, j}\right)$ with the aid of the blockwise rotation $g=g_{1} \oplus g_{2} \oplus \cdots \oplus g_{k} \oplus \cdots \in G_{1}$ :

$$
B=B_{1} \oplus \cdots \oplus B_{k} \oplus \cdots, \quad B_{k}=g_{k} A_{k} g_{k}^{-1}, \quad(k \geqslant 1) .
$$

The quadratic form with the matrix $B$ is denoted by $f_{g}(x)$. Then we have the following invariance theorem.

Theorem 11. Fix a blockwise rotation $g \in G_{1}$. For a point $x=$ $\left(x_{1}, \cdots, x_{n}, \cdots\right) \in E$ such that $\lim _{N \uparrow \infty}\|x\|_{N}$ exists, we have:

$$
\begin{gathered}
f_{g}(x)=f(g x), \\
\lim _{N \uparrow \infty} \frac{1}{N} \sum_{i, j=1}^{N} b_{i, j} x_{i} x_{j}=\lim _{N \uparrow \infty} \frac{1}{N} \sum_{i, j=1}^{N} a_{i, j}(g x)_{i}(g x)_{j} .
\end{gathered}
$$

Here (4.22) is understood in a similar manner to (4.14).

Proof. The proof is immediate.

Q.E.D.

\section{§5. Mean curvature $K$}

As stated in the introduction, the mean curvature $K$ of a surface 
$S=\{x \in D ; u(x)=0\}$ defined by a function $u(x)$ on a domain $D$ would be of the following form:

$$
K=\triangle_{\infty} u /\|\operatorname{grad} u\|_{\infty} .
$$

Here $\triangle_{\infty}$ denotes the infinite dimensional Laplacian on the space $\boldsymbol{E}$. Since the Laplacian $\triangle_{\infty}$ is thought of as twice the infinitesimal generator of the Brownian motion $B$, the part $\triangle_{\infty} u$ can be calculated rather easily with the aid of $\boldsymbol{B}$. For instance, we consider the quadratic form $u(x)=\|x\|_{\infty}^{2}$. Then we have:

$$
\|x+B(t, \omega)\|_{\infty}^{2}=\|x\|_{\infty}^{2}+t \quad \text { for all } t \geqslant 0 \text { a.s. } P^{0},
$$

which shows $\triangle_{\infty} u=2$. But the gradient part of the function $\|x+B(t, \omega)\|_{\infty}^{2}$ vanishes because of the strong law of large numbers. Consequently our next task is to define $\operatorname{grad} u(x)$ and $\|\operatorname{grad} u(x)\|_{\infty}$ analytically.

Definition 11. A \|\|$_{\infty}$-continuous function $u(x)$ on a \|\|$_{\infty}$-open set $D(\subset E)$ is said to be Fréchet differentiable at $x=a \in D$, if there exists a \|\|$_{\infty}$-continuous linear functional $\partial u(a)$ on $E$ such that

$$
u(a+h)=u(a)+(\partial u(a))(h)+o\left(\|h\|_{\infty}\right) \quad \text { for } h \in E \text { with } a+h \in D .
$$

Then it seems reasonable to define $\operatorname{grad} u$ and $\|\operatorname{grad} u\|_{\infty}$ as follows:

$(\operatorname{grad} u)_{x=a}=\partial u(a)$ and $\left\|(\operatorname{grad} u)_{x=a}\right\|_{\infty}=\sup \left\{(\partial u(a))(h) ;\|h\|_{\infty}=1\right\}$, provided that $u(x)$ is Fréchet differentiable at $x=a \in D$.

Unfortunately even the most basic quadratic form $u(x)=\|x\|_{\infty}^{2}$ is not Fréchet differentiable at some points of $\boldsymbol{E}$.

THEOREM 12. Let $\left\{N_{k} ; k \geqslant 1\right\}$ be a strictly increasing sequence of positive integers $N_{k}$ with $\lim _{k \uparrow \infty}\left(N_{1}+\cdots+N_{k-1}\right) / N_{k}=0$, and define $a$ point $a=\left(a_{1}, \cdots, a_{n}, \cdots\right) \in E$ by

$$
a_{n}= \begin{cases}\sqrt{N_{k}} & \text { if } n=N_{k}, \\ 0 & \text { otherwise } .\end{cases}
$$

Then $\|a\|_{\infty}=1$ and the quadratic form $u(x)=\|x\|_{\infty}^{2}$ on $E$ is not Fréchet differentiable at $x=a \in \boldsymbol{E}$.

Proof. First we define a point $\xi=\left(\xi_{1}, \cdots, \xi_{n}, \cdots\right) \in \boldsymbol{E}$ by

$$
\xi_{n}=\left\{\begin{array}{cl}
\sqrt{N_{2 k}} & \text { if } n=N_{2 k}, \\
-\sqrt{N_{2 k-1}} & \text { if } n=N_{2 k-1}, \\
0 & \text { otherwise. }
\end{array}\right.
$$


Then, observing $\lim _{p \uparrow_{\infty} \infty}\|a\|_{N_{p}}=\lim _{p \nmid \infty}\|\xi\|_{N_{p}}=1$, we have, $(t \in \boldsymbol{R})$ :

$$
\begin{aligned}
\|a+t \xi\|_{\infty}^{2}=\varlimsup_{N \uparrow \infty} \frac{N_{p}}{N}\left(\|a\|_{N_{p}}^{2}+t^{2}\|\xi\|_{N_{p}}^{2}+\frac{2 t}{N_{p}}\left(a_{N_{1}} \xi_{N_{1}}+\cdots+a_{N_{p}} \xi_{N_{p}}\right)\right), \\
\quad\left(N_{p} \leqslant N<N_{p+1}\right), \\
=\varlimsup_{p \uparrow \infty}\left(\|a\|_{N_{p}}^{2}+t^{2}\|\xi\|_{N_{p}}^{2}+{ }_{N_{p}}^{2 t}\left(a_{N_{1}} \xi_{N_{1}}+\cdots+a_{N_{p}} \xi_{N_{p}}\right)\right)=1+t^{2}+2|t| .
\end{aligned}
$$

Hence it holds that

$$
\lim _{t \downarrow 0}\left(\|a+t \xi\|_{\infty}^{2}-\|a\|_{\infty}^{2}\right) / t=2, \quad \lim _{t \uparrow 0}\left(\|a+t \xi\|_{\infty}^{2}-\|a\|_{\infty}^{2}\right) / t=-2,
$$

which shows our assertion.

Q.E.D.

Acknowledgement. The author would like to express his hearty thanks to Professors Takeyuki Hida, Izumi Kubo, Akinobu Shimizu and Akihiro Tsuchiya for their invaluable advices and encouragements.

\section{REFERENCES}

[1] Paul Lévy, Problèmes concrets d'analyse fonctionnelle, Gauthier-Villars, Paris, 1951.

[2] E. Bombieri, Theory of minimal surfaces and a counter-example to the Bernstein conjecture in high dimensions, The Courant Institute, New York Univ., 1970.

[3] Y. Hasegawa, Lévy's functional analysis in terms of an infinite dimensional Brownian motion I, Osaka J. Math., 19 (1982), 405-428.

[4] - Lévy's functional analysis in terms of an infinite dimensional Brownian motion II, Osaka J. Math., 19 (1982), 549-570.

[5] S. C. Port and C. J. Stone, Brownian motion and classical potential theory, Academic Press, New York, (1978).

Department of Mathematics

Nagoya Institute of Technology

Gokiso, Showa-ku, Nagoya 466, Japan 